\section{Kidney \\ Blood Pressure Research}

Review

This is an Open Access article licensed under the terms of the Creative Commons Attribution-

\title{
Performance Assessment of Patient on Dialysis
}

\author{
Fabio Manfredinia, Nicola Lambertia \\ aDepartment of Biomedical and Specialty Surgical Sciences - Section of Sport Sciences, University of \\ Ferrara; ${ }^{b}$ Department of Rehabilitation Medicine - Ferrara Universitary Hospital, Ferrara, Italy
}

Key Words

Dialysis • Exercise tests $•$ Physical activity • Exercise

\begin{abstract}
Patients on dialysis are poorly active and show a low level of physical functioning. Questionnaires and objective measurements of spontaneous physical activity are available as well as complex-expensive or simple-unexpensive tests useful to assess the patient's exercise capacity. Performance assessment unravels patients' capabilities, enables a tailored exercise prescription and provides predictive information on main clinical outcomes and therefore this topic should be of interest for nephrologists. A routinary minimal pool of tests might be usefully performed in a dialysis centre to stratify the patient's risk and to recognize patients in need of exercise training in order to address them to community-based or rehabilitative programs.
\end{abstract}

Copyright (C) 2014 S. Karger AG, Basel

\section{Introduction}

The burden of physical inactivity and the low level of physical functioning among patients affected by chronic kidney diseases and dialysis in particular, are well-known multifactorial and multinational problems [1-4]. Therefore physical activity level and physical functioning assessment, performed with valid instruments, should be a topic of specific interest in Nephrology. These measures gather information about the spontaneous activities practiced by the patient shading light on its lifestyle and attitudes on active living. These measures also assess patient's exercise capacity and physical functioning, obtain parameters useful to prescribe a personalized exercise program and allow to evaluate its effects. The patient's physical activity level can be estimated by administered or self-administered questionnaires or quantified by objective measurements, e.g. number of steps or movements performed by 


\section{Kidney Blood Pressure Research}

patients in one or more days, by means of devices such as pedometers, accelerometers, or GPS. Several questionnaires evaluating the physical activity level are available in elderly and chronically ill populations [5, 6]. Among dialysis patients the Stanford 7-day Physical Activity Recall questionnaire, the Physical Activity Scale for the Elderly, the Human Activity Profile, and the Medical Outcomes Study Short Form 36-item questionnaire have been tested and validated by comparison of the results obtained with objective measures of mobility and of physical performance [6]. The kidney disease quality of life or KDQOL questionnaire is also extensively used [7]. The direct assessment of physical activity allows to directly measure body movements, frequency and/or intensity of the activities almost without influencing the subject. Among dialysis patients these measurements have shown a number of daily steps lower than reference values for age and sex, a condition generally worsening on the non-dialysis days [8-11]. Moreover, patients' performance can be directly assessed by valid, objective and repeatable tests. They can be single, at submaximal or maximal intensity protocol, or combined to constitute a battery of tests. Besides clinical and diagnostic informations a test primarily allows to measure the exercise capacity, assigning a numeric value to the marker representing the physical quality under evaluation. In individuals with kidney disease and on dialysis [1] the tests results can be useful to compare the patients' performance to a reference value, to obtain a patients' stratification, to give information useful for the exercise training prescription, to determine the outcome of interventions as well to obtain predictive informations on relevant clinical outcomes as disability, loss of independence, hospitalization, survival, morbidity and quality of life. Exercise capacity assessment is both safe and feasible in dialysis patients [1] when considering specific cautions (e.g. number, duration and intensity of tests) related to deconditioned patients at early fatigability and high cardiovascular risk. However, costs for instruments and personnel are also factor that address the test selection. For these reasons highly reliable measures of cardiovascular fitness [1], as the cardiorespiratory test performed by measures of gas exchange during incremental treadmill or cycle ergometer protocols, as well as effective measures of muscle function by means of dynamic isotonic, isometric and isokinetic tests, are not extensively performed in patients with renal failure. Contrarily, patients are more frequently tested by simple inexpensive but effective measures of performance based on tasks required in daily living including walking tests to evaluate patients' mobility (6-minute walk test, gait speed tests), sit-to-stand tests determining muscle power or endurance of muscle legs (5-STS, 10-STS, STS-60) or tests combining different tasks (e.g. North Staffordshire Royal Infirmary Walk) [1, 12-14].

All these issues related to the exercise capacity assessment have been considered when planning the study Excite [15]. The KDQOL questionnaire was selected for measuring the quality of life of patients. Tests were selected aiming both to assess the functional outcome and to obtain as much information as possible on physical functioning, to be able to stratify patients according to their functional capacity and prescribe them semi-personalized training programs. The tests had to be performed at submaximal intensity to limit patient's fatigue. They also had to be simple, feasible and reproducible in the different venues, at low cost and not requiring instruments (e.g. ergometers). The test selected should also require a limited time of execution to minimize the number of testing days and the discomfort inside the dialysis centres. The final battery included only two tests for measuring physical functioning, the 6-minute walk test (6MWT) to evaluate mobility, with additional gait speed analysis at self-selected pace, and the 5-STS for measuring muscle power of lower limbs. By means of these tests the study allowed to take a picture of the physical functioning in a wide population of patients able to walk from dialysis centres distributed over the national territory, from Milan to Sicily. In the final phase of the study Excite, at follow up after 36 months, we also aimed to identify a questionnaire useful to evaluate the physical activity level of patients and to indirectly gather information on the level of retention of the exercise program. The choice of the questionnaire followed the same philosophy of the test selection. It had to be 


\section{Kidney \\ Blood Pressure Research}

a fast and easy tool, able to give a score and usable on wide population. We opted for the Rapid Assessment of Physical Activity (RAPA) questionnaire, based on 7 items evaluating the physical activity level and 2 items for strength and flexibility [16]. The questionnaire was translated into Italian, made of easy use for the operator by a friendly layout and completed by an additional four-item section created by us to explore patient's attitude and necessities in relation to physical activity.

\section{Conclusion}

The physical capacity assessment of patients on dialysis should be a part of their routine management $[1,14]$ but is scarcely performed. In the study Excite the tests allowed to determine a functional improvement among dialysis patients after the execution of a sixmonth exercise program [15]. As additional result the study has defined a minimal pool of tests usable in a dialysis centre, e.g. at the entry of a new patient, and able to identify at low cost, low fatigue for the patients and in a relatively short time (less than 15 minutes) relevant information as patient's mobility, power of the lower limbs, habitual level of physical activity and attitude towards it. This routinary assessment of patients might allow to stratify the patient's risk (survival, hospitalizations, peripheral disease complications, etc.), to recognize patients interested to receive counselling in relation to the benefits of exercise, to address them to simple home exercise sessions [17] or to community-based programs when available, or to specific rehabilitative programs when necessary.

\section{Disclosure Statement}

The authors of this article state that they have not any conflicts.

\section{References}

1 Koufaki P, Mercer TH: Exercise Assessment for People with End-stage Renal Failure; in Winter E, Jones A, Davison R, Bromley P and Mercer TH (eds): Sport and Exercise Physiology Testing Guidelines: The British Association of Sport and Exercise Sciences Guide Volume II. London, Routledge, 2006, chapter 20, pp. 311 326.

-2 Johansen KL, Chertow GM, Ng AV, Mulligan K, Carey S, Schoenfeld PY, Kent-Braun JA: Physical activity levels in patients on hemodialysis and healthy sedentary controls. Kidney Int 2000;57:2564-2570.

-3 Tentori F, Elder SJ, Thumma J, Pisoni RL, Bommer J, Fissell RB, Fukuhara S, Jadoul M, Keen ML, Saran R, Ramirez SP, Robinson BM: Physical exercise among participants in the Dialysis Outcomes and Practice Patterns Study (DOPPS): correlates and associated outcomes. Nephrol Dial Transplant 2010;25:3050-3062.

4 Manfredini F, Mallamaci F, Catizone L, Zoccali C: The burden of physical inactivity in chronic kidney disease: is there an exit strategy? Nephrol Dial Transplant 2012;27:2143-2145.

5 Williams K, Frei A, Vetsch A, Dobbels F, Puhan MA, Rüdell K: Patient-reported physical activity questionnaires: a systematic review of content and format. Health Qual Life Outcomes 2012;13:10-28.

6 Johansen KL, Painter P, Kent-Braun JA, Ng AV, Carey S, Da Silva M, Chertow GM: Validation of questionnaires to estimate physical activity and functioning in end-stage renal disease. Kidney Int 2001;59:1121-1127.

7 Hays RD, Kallich JD, Mapes DL, Coons SJ, Carter WB: Development of the kidney disease quality of life (KDQOL) instrument. Qual Life Res 1994;3:329-338.

-8 Zamojska S, Szklarek M, Niewodniczy M, Nowicki M: Correlates of habitual physical activity in chronic haemodialysis patients. Nephrol Dial Transplant 2006;21:1323-1327.

-9 Tudor-Locke C, Washington TL, Hart TL: Expected values for steps/day in special populations. Prev Med 2009;1:3-11. 


\section{Kidney \\ Blood Pressure Research}

10 Nowicki M, Murlikiewicz K, Jagodzińska M: Pedometers as a means to increase spontaneous physical activity in chronic hemodialysis patients. J Nephrol 2010;23:297-305.

11 Avesani CM, Trolonge S, Deléaval P, Baria F, Mafra D, Faxén-Irving G, Chauveau P, Teta D, Kamimura MA, Cuppari L, Chan M, Heimbürger O, Fouque D: Physical activity and energy expenditure in haemodialysis patients: an international survey. Nephrol Dial Transplant 2012;27:2430-2434.

-12 Mercer TH, Naish PF, Gleeson NP, Wilcock JE, Crawford C: Development of a walking test for the assessment of functional capacity in non-anaemic maintenance dialysis patients. Nephrol Dial Transplant 1998;13:2023-2026.

13 Painter P, Carlson L, Carey S, Paul SM, Myll J: Physical functioning and health-related quality-of-life changes with exercise training in hemodialysis patients. Am J Kidney Dis 2000;35:482-492.

14 Johansen KL, Chertow GM, da Silva M, Carey S, Painter P: Determinants of physical performance in ambulatory patients on hemodialysis. Kidney Int 2001;60:1586-1591.

15 Manfredini F, Bolignano D, Rastelli S, Barillà A, Bertoli S, Ciurlino D, Messa P, Fabrizi F, Zuccalà A, Rapanà R, Fatuzzo P, Rapisarda F, Bonanno G, Lombardi L, De Paola L, Cupisti A, Fuiano G, Lucisano G, Tripepi G, Mallamaci F, Catizone L, Zoccali C: Low Intensity, Home-based exercise improves physical capacity in dialysis patients: the Excite Study (Exercise introduction to enhance performance in dialysis). Nephrol Dial Transplant 2012;27:ii125

16 Topolski TD, LeGerfo J, Patrick DL, Williams B, Walwick J, Patrick MB: The Rapid Assessment of Physical Activity (RAPA) among older adults. Prev Chronic Dis 2006;3:A118.

17 Malagoni AM, Catizone L, Zamboni P, Soffritti S, Mandini S, Manfredini R, Boari B, Basaglia N, Russo G, Manfredini F: Physical capacity and quality of life perception in dialysis patients: acute and long-term effects of an exercise program prescribed at hospital-carried out at home. J Nephrol 2008;21:871-878. 